Georgian Mathematical Journal

Volume 13 (2006), Number 2, 285-290

\title{
ON DECOMPOSITIONS OF A CUBE INTO CUBES AND SIMPLEXES
}

\author{
ALEXANDER KHARAZISHVILI
}

\begin{abstract}
Some combinatorial results concerning finite decompositions (dissections) of a $k$-dimensional cube into cubes (respectively, simplexes) of the same dimension are presented in the paper. In connection with such decompositions, the notion of a decomposability number is introduced and the problem of description of all these numbers is discussed.
\end{abstract}

2000 Mathematics Subject Classification: 52A15, 52B05, 52B10.

Key words and phrases: Cube, simplex, finite decomposition, decomposability number, convex polyhedron, primitive extension, primitive polyhedron.

Let $C$ be an arbitrary $k$-dimensional cube in the Euclidean space $R^{k}$, where $k \geq 1$, and suppose that $C$ is decomposed into finitely many cubes $\left\{C_{i}: i \in I\right\}$ of the same dimension. Obviously, the edges of each cube $C_{i}$ of this decomposition are parallel to the corresponding edges of $C$. Let us denote by $N(k)$ the set of all natural numbers card $(I)$, where $\left\{C_{i}: i \in I\right\}$ ranges over all possible finite decompositions of $C$ into cubes. Clearly, the set $N(k)$ does not depend on the choice of $C$.

If $n \in N(k)$, then we say that $n$ is a decomposability number for $C$ (with respect to the family of all cubes in $R^{k}$ ). We are going to show that almost all natural numbers are decomposability numbers for $C$, i.e. there exists a natural number $r$ such that

$$
\{r+1, r+2, r+3, \ldots\} \subset N(k) .
$$

In other words, for any dimension $k \geq 1$, the set $N(k)$ is co-finite in the set $N$ of all natural numbers.

We need several simple lemmas.

Lemma 1. The following two assertions are valid:

1) $1 \in N(k)$;

2) if $a$ and $b$ are strictly positive natural numbers such that $a \geq b$ and $n \in$ $N(k)$, then $n+a^{k}-b^{k} \in N(k)$.

Proof. Assertion 1) is trivial. Let us show the validity of assertion 2). For this purpose, decompose $C$ into $a^{k}$ smaller pairwise congruent cubes and replace some $b^{k}$ cubes of them by one cube $C^{\prime}$ (this procedure is evidently possible). Since $n \in N(k)$, there exists a decomposition of $C^{\prime}$ into $n$ cubes. These $n$ cubes together with $a^{k}-b^{k}$ smaller cubes yield a decomposition of the original cube $C$ into $n+a^{k}-b^{k}$ cubes. Thus, we obtain the required relation $n+a^{k}-b^{k} \in$ $N(k)$. 
Lemma 2. Suppose that natural numbers

$$
a_{1}, a_{2}, \ldots, a_{p}, b_{1}, b_{2}, \ldots, b_{p}
$$

satisfying the inequalities

$$
a_{1} \geq b_{1}, a_{2} \geq b_{2}, \ldots, a_{p} \geq b_{p}
$$

are given and suppose that $n \in N(k)$. Then we have

$$
n+\left(a_{1}^{k}-b_{1}^{k}\right)+\left(a_{2}^{k}-b_{2}^{k}\right)+\cdots+\left(a_{p}^{k}-b_{p}^{k}\right) \in N(k) .
$$

Proof. This lemma easily follows from Lemma 1 by using the induction on $p$.

Remark 1 . Let us denote $d=2^{k}-1$. In virtue of Lemma 2 , we can deduce that if $n \in N(k)$ and $p$ is a natural number, then $n+p d \in N(k)$. Indeed, it suffices to put in the above-mentioned lemma

$$
\begin{gathered}
a_{1}=a_{2}=\cdots=a_{p}=2, \\
b_{1}=b_{2}=\cdots=b_{p}=1 .
\end{gathered}
$$

Lemma 3. Let $j$ be an arbitrary element of the set $\{0,1, \ldots, d-1\}$, where $d$ is as in Remark 1. Then there exists a number $n_{j} \in N(k)$ such that

$$
n_{j}=t_{j} d+j
$$

for some positive integer $t_{j}$.

Proof. Taking into account Lemmas 1 and 2, we may write

$$
a_{1}^{k}+a_{2}^{k}+\cdots+a_{p}^{k}-(p-1) \in N(k)
$$

for any finite sequence $\left(a_{1}, a_{2}, \ldots, a_{p}\right)$ of natural numbers satisfying the inequalities

Let us define

$$
1 \leq a_{1}, 1 \leq a_{2}, \ldots, 1 \leq a_{p}
$$

$$
p=d-j+1, a_{1}=a_{2}=\cdots=a_{p}=d .
$$

It can be easily verified that in this case we have

$$
a_{1}^{k}+a_{2}^{k}+\cdots+a_{p}^{k}-(p-1)=t_{j} d+j
$$

for an appropriate positive integer $t_{j}$, which completes the proof.

Theorem 1. There exists a natural number $r$ such that

$$
\{r+1, r+2, r+3, \ldots\} \subset N(k) .
$$

Proof. According to the previous lemma, for each $j \in\{0,1, \ldots, d-1\}$, there exists a number $n_{j} \in N(k)$ satisfying the equality

$$
n_{j}=t_{j} d+j
$$

for some positive integer $t_{j}$. Let us put

$$
r=\max \left(n_{0}, n_{1}, \ldots, n_{d-1}\right)
$$

and show that $r$ is the required natural number. 
Indeed, take any natural number $n>r$. Clearly, $n$ can be represented in the form

$$
n=t d+j,
$$

where $t$ is a positive integer and $j \in\{0,1, \ldots, d-1\}$. Consequently, we get

$$
n-n_{j}=\left(t-t_{j}\right) d
$$

or, equivalently,

$$
n=n_{j}+\left(t-t_{j}\right) d .
$$

Since $n_{j} \in N(k)$, we finally have $n \in N(k)$ (in view of Remark 1 ). The proof of Theorem 1 is completed.

Remark 2. In general (i.e. for an arbitrary dimension $k$ ), a complete description of the set $N(k)$ is unknown. Some more detailed information about $N(k)$ can be obtained for small natural numbers $k$. Obviously,

$$
N(1)=\{1,2,3, \ldots, n, \ldots\} .
$$

Also, it can be easily verified that

$$
N(2)=\{1,4,6,7,8, \ldots, n, \ldots\}
$$

and

$$
\{71,72,73, \ldots, n, \ldots\} \subset N(3) .
$$

In this context, it should also be mentioned that none of numbers $n$ satisfying the inequalities $1<n<2^{k}$ belongs to the set $N(k)$. In addition, we have $2^{k} \in N(k)$ but $2^{k}+1 \notin N(k)$ for $k \geq 2$.

Remark 3. A square can be decomposed into finitely many squares whose sizes are pairwise distinct. This result was first obtained by A. Stöhr and R. Sprague (see [1] and [2]). At the present time we know of the constructions which yield a decomposition of a square into 24 squares with pairwise distinct sizes. On the other hand, it is not difficult to prove that, for $k \geq 3$, none of the $k$-dimensional cubes can be decomposed into finitely many $k$-dimensional cubes whose sizes differ from each other (for more details about this topic see, e.g., [3]).

Let $P$ be a $k$-dimensional rectangular parallelepiped in the Euclidean space $R^{k}$. We denote by $N_{P}$ the set of all natural numbers $n$ for which there exists at least one decomposition of $P$ into $n$ cubes of dimension $k$. Obviously, if $P$ is a $k$-dimensional cube, then the set $N_{P}$ coincides with the set $N(k)$ introduced earlier. If $n \in N_{P}$, then we say that $n$ is a decomposability number for $P$ (with respect to the family of all cubes in $R^{k}$ ).

Theorem 2. For any rectangular parallelepiped $P$, the set $N_{P}$ is either empty or co-finite in $N$.

Proof. If $N_{P}=\varnothing$, then there is nothing to prove.

Suppose now that $N_{P} \neq \varnothing$ and consider a finite decomposition $\left\{C_{i}: i \in\right.$ $I\}$ of $P$ into cubes. Fix some cube $C_{i}$ of this decomposition. According to Theorem 1, there exists a natural number $r$ such that all elements of the set 
$\{r+1, r+2, r+3, \ldots\}$ are decomposability numbers for $C_{i}$. Now, it is clear that all elements of the set

$$
\{\operatorname{card}(I)+r, \operatorname{card}(I)+r+1, \operatorname{card}(I)+r+2, \ldots\}
$$

are decomposability numbers for $P$. This ends the proof of Theorem 2 .

Remark 4. Denote by $l_{1}, l_{2}, \ldots, l_{k}$ the lengths of all edges of a given $k$ dimensional rectangular parallelepiped $P$, passing through one of its vertices. As shown by Dehn, the set $N_{P}$ is nonempty if and only if all fractions

$$
l_{1} / l_{2}, l_{2} / l_{3}, \ldots, l_{k-1} / l_{k}
$$

are rational numbers (in this connection, see, e.g., [4]).

Consider again a $k$-dimensional cube $C$ in the space $R^{k}$, where $k \geq 1$. Let $\left\{T_{i}: i \in I\right\}$ be a finite decomposition of $C$ into simplexes of the same dimension. We say that $m=\operatorname{card}(I)$ is a decomposability number for $C$ (with respect to the family of all simplexes in $R^{k}$ ). The set of all such $m$ is denoted by $M(k)$. Observe that

$$
M(k)=\{s, s+1, s+2, \ldots\},
$$

where $s=s_{C}=s(k)$ is the smallest number of simplexes into which the cube $C$ can be decomposed. Therefore the problem of description of the set $M(k)$ is equivalent to the problem of finding the precise values of the function $s(k)(k=$ $1,2,3, \ldots)$.

Remark 5. Clearly, we have $s(1)=1, s(2)=2$ and $s(3)=5$. By using a combinatorial argument based on some upper estimates of the volumes of the simplexes contained in a given cube, it is not difficult to show that $s(4) \geq 13$. This simple inequality will be applied below (see Theorem 3).

More generally, for a given $k$-dimensional convex polyhedron $Q$ in the space $R^{k}$, denote by $s_{Q}$ the smallest number of simplexes into which $Q$ can be decomposed. The values of the function $s_{Q}$ essentially depend on the combinatorial structure of $Q$.

Let $Q$ and $Q^{\prime}$ be two $k$-dimensional convex polyhedra in $R^{k}$. We say that $Q^{\prime}$ is a primitive extension of $Q$ if there exists a $k$-dimensional simplex $T$ in $R^{k}$ such that:

(1) $Q \cap T$ is a common facet of $Q$ and $T$;

(2) the set of vertices of $Q^{\prime}$ is the union of the sets of vertices of $Q$ and $T$.

It immediately follows from conditions (1) and (2) that $Q^{\prime}=Q \cup T$.

Accordingly, we say that a $k$-dimensional convex polyhedron $Q$ is primitive if there exists a finite sequence $\left\{Q_{1}, Q_{2}, \ldots, Q_{n}\right\}$ of convex polyhedra in $R^{k}$ such that:

(a) $Q_{1}$ is a $k$-dimensional simplex in $R^{k}$;

(b) for each integer $i \in[1, n-1]$, the polyhedron $Q_{i+1}$ is a primitive extension of $Q_{i}$;

(c) $Q_{n}=Q$. 
Remark 6. Any convex polygon in the plane $R^{2}$ is primitive in the sense of the above definition. Actually, an analogous fact is true for any simple polygon in $R^{2}$ (see, e.g., [5] where some closely related results are also presented).

A three-dimensional cube $C$ is primitive (this fact is closely connected with the equality $s(3)=5$ and is of some interest from the purely geometrical viewpoint because no facet of $C$ is a triangle).

But the most convex polyhedra in the space $R^{3}$ are not primitive. In particular, if a convex three-dimensional polyhedron $Q$ has no trihedral angle, then $Q$ cannot be primitive (cf. also Example 1 below).

Lemma 4. Let $Q$ be a three-dimensional convex polyhedron in $R^{3}$ and let $v=v(Q)$ denote the number of vertices of $Q$. The following assertions are valid:

1) $s_{Q} \geq v-3$;

2) $s_{Q}=v-3$ if and only if $Q$ is primitive.

The proof of this lemma is based on the classical Euler formula

$$
v+f=e+2
$$

and on the elementary fact that, for every convex polygon $P \subset R^{2}$ with $n$ sides, the minimal number of triangles into which $P$ can be decomposed is equal to $n-2$ (this fact does not hold for nonconvex polygons).

It directly follows from Lemma 4 that if $Q$ is a three-dimensional convex polyhedron and $s_{Q} \geq v-2$, then $Q$ is not primitive. We also claim that $s_{Q}$ cannot be represented as a function of a single variable $v=v(Q)$.

Note that the convexity of $Q$ is essential in the formulation of Lemma 4. Indeed, if $Q$ is an arbitrary three-dimensional polyhedron in $R^{3}$, then the inequality $v(Q) \leq 4 s_{Q}$ holds true. At the same time, for any natural number $n \geq 1$, there exists a three-dimensional simple polyhedron $Q$ such that $v(Q)=4 n$ and $s_{Q}=n$.

Obviously, analogous facts are valid for $k$-dimensional polyhedra in the space $R^{k}(k \geq 2)$, where we have the inequality $v(Q) \leq(k+1) s_{Q}$ and, for any natural number $n \geq 1$, there exists a $k$-dimensional simple polyhedron $Q$ such that $v(Q)=(k+1) n$ and $s_{Q}=n$.

Example 1. In the space $R^{3}$ consider a convex bipyramid $Q$ with $2 n+2$ vertices, where $n \geq 2$. It is easy to see that there are some $2 n$ faces $F_{1}, F_{2}, \ldots, F_{2 n}$ of $Q$ such that the intersection $F_{i} \cap F_{j}$ either is empty or is a singleton for any two distinct integers $i$ and $j$ from the set $\{1,2, \ldots, 2 n\}$. This fact implies that every decomposition of $Q$ into tetrahedra needs at least $2 n$ members. Consequently, we come to the inequality

$$
s_{Q} \geq 2 n=(2 n+2)-2 .
$$

In particular, this inequality shows that the bipyramid $Q$ is not primitive. The latter fact is trivial, since $Q$ has no trihedral angles. Let $Q^{\prime}$ denote a primitive extension of $Q$. Then $Q^{\prime}$ has a trihedral angle but is not primitive either. 
Note that a simplicial decomposition of $Q$ into exactly $2 n$ tetrahedra can be constructed without any difficulty.

Example 2. According to the preceding example, a convex hexagonal bipyramid $Q$ in the space $R^{3}$ needs at least 6 tetrahedra for its decomposition, i.e. $s_{Q}=6$. The number of vertices of $Q$ is equal to 8 . A three-dimensional cube $C$ has the same number of vertices. However, $s_{C}=s(3)=5$ (cf. Remarks 5 and 6 ). We see again that, for a general three-dimensional convex polyhedron $P$ in the space $R^{3}$, the value $s_{P}$ cannot be represented as a function of a single variable $v=v(P)$.

Theorem 3. A four-dimensional cube $C$ is not primitive.

Proof. From the definition of a primitive $k$-dimensional convex polyhedron $Q$ it follows (by easy induction) that $s_{Q} \leq v(Q)-k$, where $v(Q)$ denotes the number of vertices of $Q$.

Now, suppose to the contrary that $C$ is primitive. Then we must have

$$
s_{C} \leq v(C)-4=16-4=12 .
$$

But, as mentioned in Remark $5, s_{C}=s(4) \geq 13$. The contradiction obtained ends the proof of Theorem 3.

It readily follows from Theorem 3 that, for each natural number $k \geq 4$, the $k$-dimensional unit cube $C \subset R^{k}$ is not primitive. To show this, it suffices to apply induction on $k$ taking into account the fact that the volume of any simplex contained in $C$ does not exceed $1 / k$.

\section{REFERENCES}

1. A. Stöhr, Über Zerlegungen von Rechtecken in inkongruente Quadrate. Schr. Math. Inst. Inst. Angew. Math. Univ. Berlin 4(1939), 119-140.

2. R. Sprague, Beispiel einer Zerlegung des Quadrats in lauter verschiedene Quadrate. (German) Math. Z. 45(1939), 607-608.

3. R. L. Brooks, C. A. B. Smith, A. H. Stone, and W. T. Tutte, The dissection of rectangles into squares. Duke Math. J. 7(1940), 312-340.

4. H. HAdwiger, Vorlesungen ber Inhalt, Oberfläche und Isoperimetrie. (German) Springer-Verlag, Berlin-Göttingen-Heidelberg, 1957.

5. A. B. Kharazishvili, Simple polyhedra. (Russian) Sem. Inst. Prikl. Mat. Dokl. No. 18, (1984), 34-38, 83.

(Received 18.08.2005)

Author's Address:

A. Razmadze Mathematical Institute

Georgian Academy of Sciences

1, M. Aleksidze St., Tbilisi 0193, Georgia

I. Vekua Institute of Applied Mathematics

I. Javakhishvili Tbilisi State University,

2, University St., Tbilisi 0143, Georgia

E-mail: kharaz2@yahoo.com 